\title{
X-ray and UV long-term properties of 3C 454.3 during low gamma-ray states: the Swift view
}

\author{
Stefano Vercellone* and Patrizia Romano \\ INAF/IASF Palermo \\ E-mail: stefanodifc.inaf.it, romanodifc.inaf.it
}

3C 454.3 is one of the most investigated gamma-ray blazars. Thanks to an intensive Swift monitoring, we showed that, during extreme gamma-ray flares, the harder-when-brighter correlation noted in the X-ray energy band during intermediate gamma-ray states does not hold anymore, leading to an achromatic increase of the X-ray flux. We show here the preliminary results collected during a recent campaign (2012 November - 2014 October) whose aim is to investigate, by means of deep monthly observations, the peculiar X-ray spectral properties when the source is in a quiescent to very low gamma-ray flux level. Our observations, thanks to a precise photon index determination, allow us to investigate the $\mathrm{X}$-ray spectral properties with an unprecedented accuracy. Our results seem to confirm that, at X-ray flux levels below $2 \times 10^{-11} \mathrm{erg} \mathrm{cm}^{-2} \mathrm{~s}^{-1}(2-$ $10 \mathrm{keV}$ ), neither the harder-when-brighter, nor the achromatic trend holds anymore. Moreover, we reveal the presence of gamma-ray orphan fast optical/UV flares.

Swift: 10 Years of Discovery

2-5 December 2014

La Sapienza University, Rome, Italy

\footnotetext{
*Speaker.
} 


\section{The $\gamma$-ray Blazar $3 \mathrm{C} 454.3$}

The blazar 3C 454.3 (PKS 2251+158; $z=0.859$ ) is a well-known $\gamma$-ray flat spectrum radio quasar which exhibits high dynamic range flux variations from the radio to the $\gamma$-ray energy bands. It has been intensively studied both by AGILE (see [1] for a review of the AGILE results) and by Fermi (e.g., [2] and reference therein) during the several high-intensity gamma-ray flares since July 2007. A preliminary [3] physical modeling of the spectral energy distributions (SEDs), when 3C 454.3 was at differing flux levels, provided an interpretation of the emission mechanism responsible for the radiation emitted in the $\gamma$-ray energy band, assumed to be inverse Compton scattering of photons from the broad-line region (BLR) clouds off the relativistic electrons in the jet, with bulk Lorentz factor $\Gamma \sim 20$. More recently, the extreme intensity of the flare that occurred on 2010 November 20 (3-hour peak of $F_{E>100 \mathrm{MeV}}=(85 \pm 5) \times 10^{-6}$ photons cm $^{-2} \mathrm{~s}^{-1}$, [2]) and its large dynamic range if compared with the low $\gamma$-ray state in Winter 2008 (about a factor of 100 at $100 \mathrm{MeV}$, [4]) motivated us to study the nature and the site of the $\gamma$-ray emission (e.g., [5], [6], [7], and [8]). In particular, during extreme $\gamma$-ray flares, [9] showed that the harder-when-brighter correlation noted during low and intermediate $\gamma$-ray states (and discussed in [3]) does not hold anymore, leading to an achromatic increase of the X-ray flux. On the other hand, the quiescent $\gamma$-ray emission phase has been poorly investigated so far, and the large errors in the photon index determination at low $\left(\leq 2 \times 10^{-11} \mathrm{erg} \mathrm{cm}^{-2} \mathrm{~s}^{-1}, 2-10 \mathrm{keV}\right) \mathrm{X}$-ray fluxes do not allow us to establish a firm relation between the two quantities.

3C 454.3 represents an excellent laboratory to investigate the long-term X-ray behavior. Since its launch, Swift/XRT observed and detected this source at different flux levels. Swift/UVOT also allows us to investigate, during low activity states, the region of the SED where the signature of the accretion disc is not completely washed out by the synchrotron jet component. We present a preliminary analysis of a 2-yr Swift monitoring of 3C 454.3. A more detailed discussion of our findings will be presented in [10].

\section{The 2012-2013 and 2013-2014 Campaigns}

The lack of a high signal-to-noise spectral index determination at low X-ray fluxes motivated us to perform two Swift monitoring campaigns in order to investigate any possible spectral index X-ray flux correlations. The first campaign started on 2012 November 27, ended on 2013 October 27, for a total of $63.9 \mathrm{ks}$. The second campaign started on 2013 November 14 and ended on 2014 October 14, for a total of $55.5 \mathrm{ks}$. Our observing strategy was to observe 3C 454.3 once a month, $7 \mathrm{ks}$ per observation, for one year. With the typical count-rate during low states of $(0.1-0.2) \mathrm{cts} \mathrm{s}^{-1}$, we obtained about $1000 \mathrm{cts}^{\text {observation }}{ }^{-1}$. This allowed us to obtain a precise estimate of the X-ray spectrum $(\Delta \Gamma / \Gamma \lesssim 15 \%)$. Moreover, we obtained Swift/UVOT coverage on almost all the UV and optical filters, allowing us to simultanously monitor both the decaying portion of the synchrotron emission component and the rising wing of the inverse Compton one.

Figure 1 shows the long-term (2005-2014) Fermi/LAT $\gamma$-ray (E $>100 \mathrm{MeV}$, upper panel) and the Swift/XRT X-ray $(0.3-10 \mathrm{keV}$, lower panel) light curves, respectively. The Fermi/LAT light curve is taken from the publicly available "Monitored Source List Light Curve ${ }^{1}$ ", while the

\footnotetext{
${ }^{1}$ http://fermi.gsfc.nasa.gov/ssc/data/access/lat/msl_lc/
} 


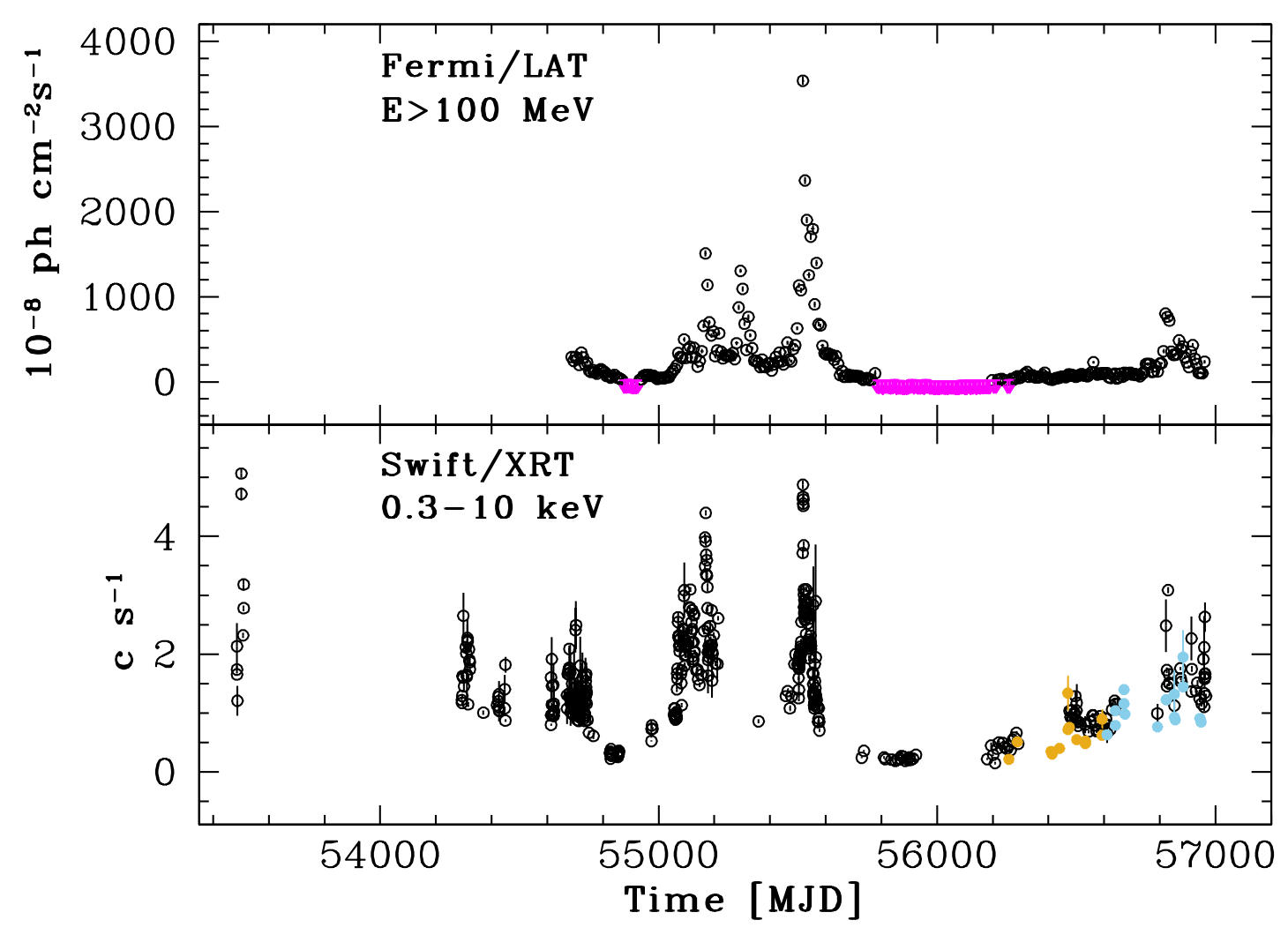

Figure 1: Long-term (2005-2014) $\gamma$-ray (upper panel: Fermi/LAT, $E>100 \mathrm{MeV}$, 1-week resolution) and X-ray (lower panel: Swift/XRT, 0.3-10 keV) 3C 454.3 light curves. Magenta points are 3- $\sigma$ upper limits. Golden and blue points represent the 2012-2013 and 2013-2014 Swift/XRT data, respectively.

Swift/XRT light curve has been produced by means of the "Swift/XRT Data Products Generator" (see [11] and references therein for a detailed description of the on-line tool). The $\gamma$-ray data are binned on a weekly time-scale, in order to better emphasize the general trend above $100 \mathrm{MeV}$. The $\mathrm{X}$-ray data are binned on the observation time-scale (typically 5-7 ksec). The 3- $\sigma \gamma$-ray upper limits (Figure 1, upper panel, magenta points) show the very low flux state which triggered our campaigns, as reported by the golden and blue points (Figure 1, lower panel).

\section{Results}

Both Swift/XRT and Swift/UVOT data were reduced and analyzed, by means of FTOOLS ( $\mathrm{V}$ 6.16), according to the standard procedures summarized in [9]. As shown in Figure 1, our monitoring campaigns started during a low or intermediate $\gamma$-ray flux state. During the 2012-2013

\footnotetext{
${ }^{2}$ http://www.swift.ac.uk/user_objects/
} 
campaign, all the X-ray points (with the exception od one in windowed time mode) where below a count rate of $1 \mathrm{cts} \mathrm{s}^{-1}$, while the 2013-2014 campaign monitored an increase of the $\gamma$-ray flux, including a short and moderate $\gamma$-ray flare $\left(F_{\mathrm{E}>100 \mathrm{MeV}} \simeq 10^{-5}\right.$ photons $\left.\mathrm{cm}^{-2} \mathrm{~s}^{-1}\right)$ during June 2014 .

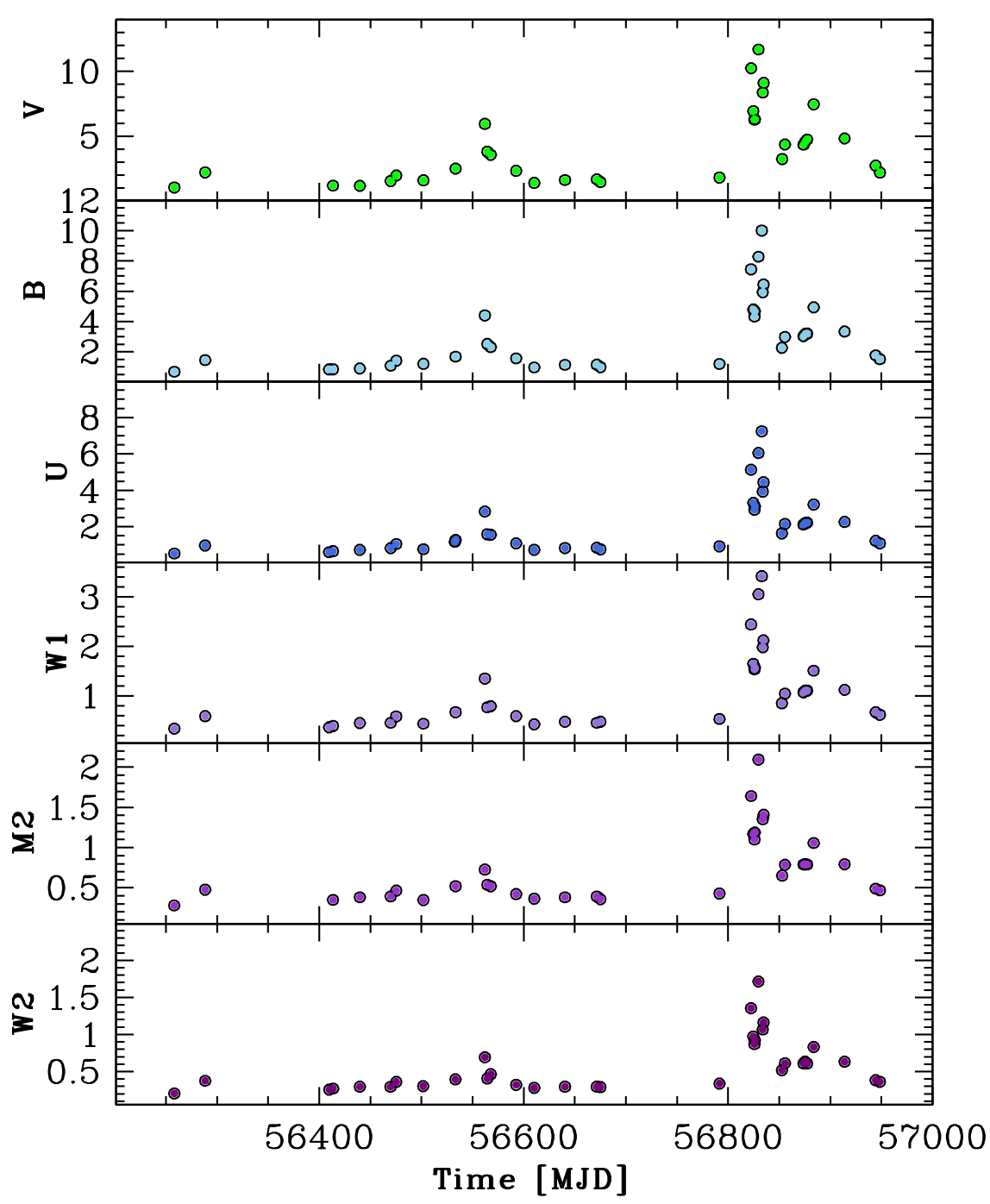

Figure 2: Swift/UVOT light curves [mJy] in different filters, covering the period 2012-11-27 to 2014-10-14.

Figure 2 shows the Swift/UVOT light curves [in mJy] in different filters, covering the period 2012-11-27 - 2014-10-14. A clear optical/UV flare is visible, starting around MJD 56822 (2014 June 14), detected both in $\gamma$-ray [12] and in X-ray. Moreover, a $\gamma$-ray orphan, fast optical/UV flare was detected on MJD 56562 (2013 September 27), as noted also in [13]. Previously, a similar behaviour was reported in [9]. A preliminary explanation of the lack of a simultaneous $\gamma$-ray flare 
requires a magnetic field enhancement by a factor of two, or a local $\gamma-\gamma$ absorption, or a locally seed-photon-starved zone. Alternatively, [5] locate the optical precursor emitting zone near the black-hole, at the broad-line region distance, down to a distance of $\simeq 10^{17} \mathrm{~cm}$, where the high magnetic fields and electron densities lead to enhanced synchrotron emission in the optical-UV. These conditions allow a jet plasmoid to produce the $\gamma$-ray orphan, fast optical/UV flare observed on MJD 56562.

The preliminary results of our monitoring campaigns are summarized in Figure 3, where we plot the X-ray photon index as a function of the (observed) $2-10 \mathrm{keV}$ flux during different observing campaigns.

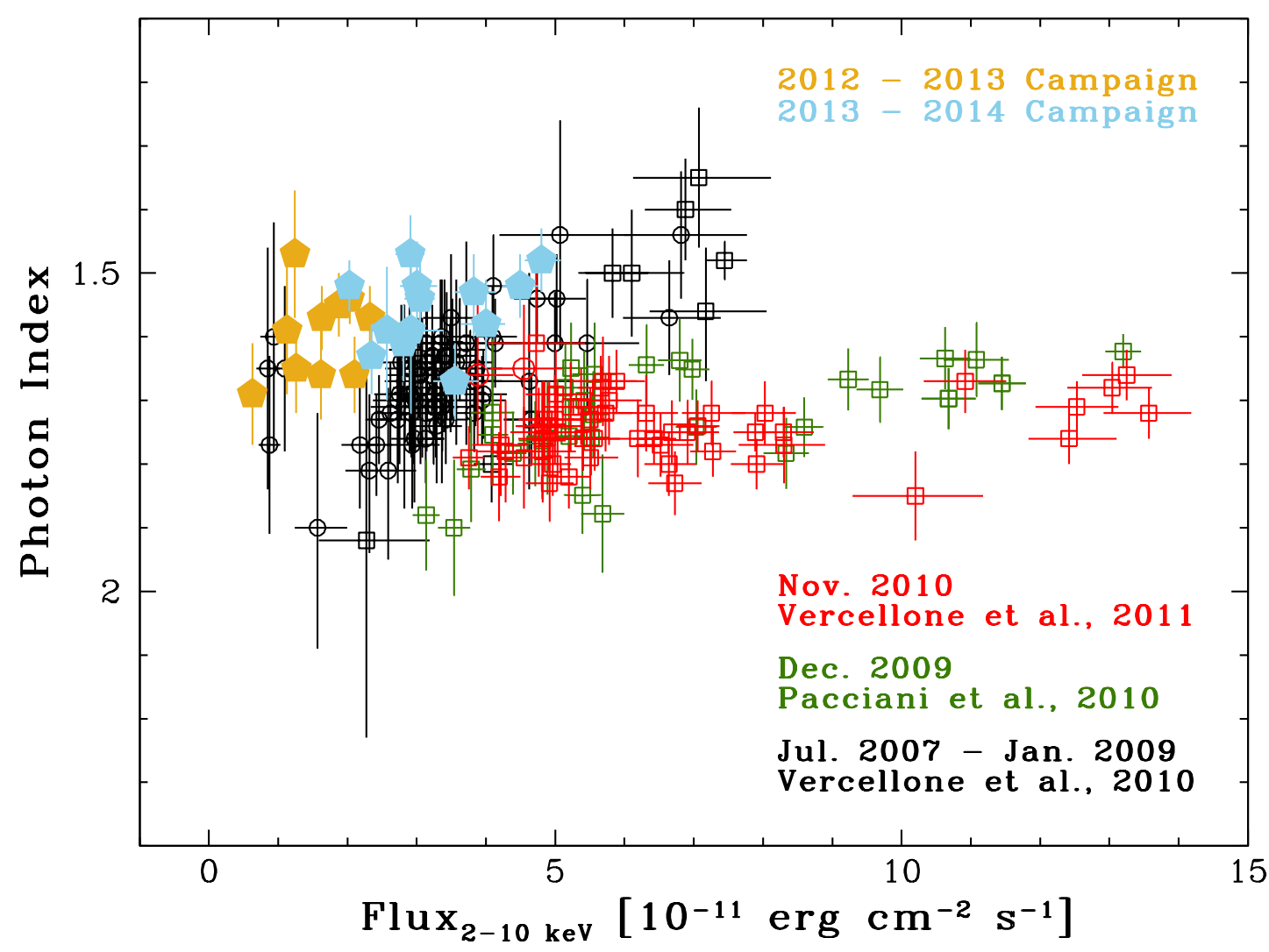

Figure 3: The Swift/XRT X-ray photon index as a function of the (observed) $2-10 \mathrm{keV}$ flux during different observing campaigns. Data from [3] (black points), [14] (green points), and [9] (red points). Golden and blue points represent the 2012-2013 and 2013-2014 data, respectively.

This allows us to investigate the photon index vs. X-ray flux correlation during both low and intermediate $\gamma$-ray flux states. In particular, we compare the 2007-2009 monitoring campaign (black points), when 3C 454.3 was in an intermediate $\gamma$-ray flux level, with the extreme $\gamma$-ray flares in 2009 (green points) and 2010 (red points). During the 18-months AGILE campaign, [3] found a clear trend, in particular for fluxes above $2 \times 10^{-11} \mathrm{erg} \mathrm{cm}^{-2} \mathrm{~s}^{-1}$. We can describe the harder- 
when-brighter trend in terms of a dominant contribution of the external Compton (EC) off the disk seed photons, EC(Disk), over the synchrotron self-Compton (SSC) component, probably due to an increase of the accretion rate. The constant X-ray photon index during the extreme $\gamma$-ray flares in 2009 and 2010 can be interpreted in terms of a balance of the SSC contribution with respect to the $\mathrm{EC}(\mathrm{Disk})$, due to the possible increase of the break energy Lorentz factor, $\gamma_{b}$, during the super-flares with respect to the value during the less energetic $\gamma$-ray flares. By increasing $\gamma_{b}$, we obtain both an increase of the EC(Disk) component (and the shift of the peak of its emission to higher frequencies), and a simultaneous increase of the SSC. The net result is a roughly achromatic increase of the X-ray emission.

The 2012-2013 data-set (golden points) shows that, for $F_{(2-10 \mathrm{keV})} \leq 2 \times 10^{-11} \mathrm{erg} \mathrm{cm}^{-2} \mathrm{~s}^{-1}$, neither the harder-when-brighter trend (black points, typical of less energetic $\gamma$-ray states) nor the quasi-achromatic increase of the X-ray flux (red and green points, occurring during extreme $\gamma$-ray flares) seem to emerge. On the other hand, the 2013-2014 data-set (blue points) collected during slightly higher $\gamma$-ray flux levels shows that the harder-when-brighter trend seems to hold. The blue points do not follow the green (2009 super-flare) and red (2010 super-flare) points which are characteristic of extreme $\gamma$-ray states. Our campaigns allow us to monitor all the different flux levels. This project shows that only by means of deep observations we can disentangle the different spectral trends, and investigate the relative contributions of the different emission mechanisms to the spectral energy distribution.

\section{Acknowledgements}

We acknowledge contract ASI-INAF I/004/11/0.

\section{References}

[1] S. Vercellone for the AGILE Team, ArXiv e-prints 1205.5510 (2012).

[2] A. A. Abdo, M. Ackermann, M. Ajello, et al., ApJL 733, L26+ (2011).

[3] S. Vercellone, F. D'Ammando, V. Vittorini, et al., ApJ 712, 405-420 (2010).

[4] S. Vercellone, International Journal of Modern Physics Conference Series 8, 404-407 (2012).

[5] V. Vittorini, M. Tavani, A. Cavaliere, E. Striani, and S. Vercellone, ApJ 793, 98 (2014).

[6] L. Pacciani, F. Tavecchio, I. Donnarumma, et al., ApJ 790, 45 (2014).

[7] K. Nalewajko, M. C. Begelman, and M. Sikora, ApJ 789, 161 (2014).

[8] G. Bonnoli, G. Ghisellini, L. Foschini, F. Tavecchio, and G. Ghirlanda, MNRAS 410, 368-380 (2011).

[9] S. Vercellone, E. Striani, V. Vittorini, et al., ApJL 736, L38+ (2011).

[10] S. Vercellone, and P. Romano, in preparation (2015).

[11] P. A. Evans, A. P. Beardmore, K. L. Page, et al., MNRAS 397, 1177-1201 (2009).

[12] A. Bulgarelli, M. Tavani, V. Fioretti, et al., The Astronomer's Telegram 6234, 1 (2014).

[13] V. M. Larionov, D. A. Morozova, N. V. Efimova, D. A. Blinov, and A. Di Paola, The Astronomer's Telegram 5423, 1 (2013).

[14] L. Pacciani, V. Vittorini, M. Tavani, et al., ApJL 716, L170-L175 (2010). 